\title{
Lack of association between Screencell-detected circulating tumour cells and long-term survival of patients undergoing surgery for non-small cell lung cancer: A pilot clinical study
}

\author{
JAMES BARR $^{1}$, DIMPLE CHUDASAMA ${ }^{1,2}$, ALEX RICE $^{1}$, EMMANOUIL KARTERIS ${ }^{2}$ and VLADIMIR ANIKIN ${ }^{1,3}$ \\ ${ }^{1}$ Department of Thoracic Surgery, Royal Brompton and Harefield NHS Foundation Trust, Harefield UB9 6JH; \\ ${ }^{2}$ Department of Biomedical Science, Brunel University, London UB8 3PH, UK; ${ }^{3}$ Department of Oncology and \\ Reconstructive Surgery of I.M. Sechenov First Moscow State Medical University, 119146 Moscow, Russia
}

Received March 31, 2019; Accepted October 29, 2019

DOI: $10.3892 / \mathrm{mco} .2020 .1981$

\begin{abstract}
Circulating tumour cells (CTCs) are cancer cells of epithelial origin that are present in peripheral blood samples. ScreenCell detection of CTCs and the association with long term survival in non-small cell lung cancer (NSCLC) patients was evaluated in the present study. A total of 33 patients undergoing surgical resection for NSCLC were recruited. Patients were followed up for 5-years post-operatively. Pre-operative patient bloods samples were processed using ScreenCell. CTCs were detected in $26(79 \%)$ patients. In patients who were positive for CTCs, a total of $9(35 \%)$ patients succumbed to the disease, whereas in patients negative for CTCs, a total of 4 (57\%) patients succumbed to the disease $(\mathrm{P}=0.29)$. No association was identified between positive CTCs and poorer survival (Chi-squared 1.47, $\mathrm{P}=0.23$; hazard ratio, $0.42 ; 95 \%$ confidence interval: $0.1-1.7)$. The presence of CTCs detected with ScreenCell does not influence prognosis in patients with NSCLC that was operated on. The high rate of CTC detection is encouraging in supporting this technology to aid early lung cancer diagnosis.
\end{abstract}

\section{Introduction}

Lung cancer remains the leading cause of cancer-related mortality amongst men and women in the United Kingdom (1). The lack of symptoms in the early disease means that three quarters of lung cancers are diagnosed at a late stage, often disqualifying patients from curative treatment (2). Screening with early diagnosis and treatment has been shown to improve survival (3).

Correspondence to: Professor Vladimir Anikin, Department of Thoracic Surgery, Royal Brompton and Harefield NHS Foundation Trust, Hill End Road, Harefield UB9 6JH, UK

E-mail: v.anikin@rbht.nhs.uk

Key words: screen cell, circulating tumour cells, lung cancer
Metastatic disease is responsible for most cancer deaths and for this to occur tumour cells must separate from the primary tumour and circulate in the bloodstream to distant sites (4). Circulating tumour cells (CTCs) are cancer cells of epithelial origin that are present in the peripheral blood samples of cancer patients. They form a subpopulation of tumour cells which intravasate to allow haematogenous dissemination to other areas of the body, contributing to metastatic spread (4). Their use includes early detection of cancers, monitoring response to treatment and assessing for reoccurrence (5).

Isolation of CTCs has been attempted by using physical features such as their larger size and weight, antibody based platforms, and microfluidic techniques, where spaces and flows are commensurate with a scale of single cells, allowing CTCs to be captured $(6,7)$. ScreenCell detection of CTCs relies on the size of the CTC and is not antibody dependent, removing the antibody-bias we see with some other techniques.

We have investigated the use of ScreenCell in operated patients with lung cancer in previous studies (8-12). In this study we evaluate the value of CTC detection and association with long term survival.

Our main outcome of interest was whether the presence of CTCs would impact long term survival. Secondary outcomes of interest included whether the presence of CTCs would correlate with the stage of the cancer and if there was a difference between histological subtype of tumour and the proportion of patients with CTCs. In addition to evaluate for any differences in the proportion of CTCs in patients undergoing a thoracoscopic or open surgical approach.

\section{Patients and methods}

A total of 33 patients undergoing surgical treatment with curative intent for non-small cell lung cancer (NSCLC) were recruited from August, 2012 to June, 2015 at Harefield Hospital (Uxbridge, UK). The median age of the patients was 66 years (range, 41-87 years) and 15 (45\%) patients were male. Patients had a confirmed diagnosis of NSCLC either pre-operatively or on an intra-operative frozen section. Ethical approval was sought prior (ethical approval no. 10/H0504/9), with consent obtained pre-operatively. Patients who 
Table I. Median survival depending on histology and CTC status.

\begin{tabular}{|c|c|c|c|c|c|c|}
\hline \multirow[b]{2}{*}{ Histology } & \multirow[b]{2}{*}{ Total } & \multicolumn{2}{|c|}{ CTC status } & \multicolumn{3}{|c|}{ Median survival (months) } \\
\hline & & Detected & Not detected & Total & CTCs detected & CTCs not detected \\
\hline Adenocarcinoma & $21(64 \%)$ & $17(81 \%)$ & $4(19 \%)$ & 38 & 38 & 31 \\
\hline $\mathrm{SqCC}$ & $12(36 \%)$ & $9(75 \%)$ & $3(25 \%)$ & 53 & 60 & 34 \\
\hline Total & 33 & $26(79 \%)$ & $7(21 \%)$ & 39 & $47^{\mathrm{a}}$ & $34^{\mathrm{a}}$ \\
\hline
\end{tabular}

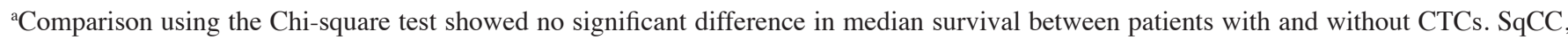
squamous cell carcinoma; CTCs, circulating tumour cells.

participated in this research had complete clinical data. The signed informed consents were obtained from the patients or the guardians. Surgery was performed under the care of four thoracic surgeons at a tertiary thoracic centre. Operations were performed via a thoracotomy or via video assisted thoracotomy surgery (VATS) approach. One case was performed via a sternotomy where there were bilateral lung lesions.

Patients were followed-up for a median time period of 5 years post-operatively. Survival information was obtained by contacting the patient's General Practitioner in January, 2018.

The detailed technique of CTC detection using the ScreenCell device is described elsewhere (9). Briefly, three millilitres of blood was collected from the peripheral vein of patients immediately prior to surgery in EDTA tubes. Samples were incubated with a lysis and formaldehyde fixation buffer provided by ScreenCell. Samples were then filtered through the ScreenCell ${ }^{\circledR}$ device as per the manufacturer's protocol.

Post-filtration filters were removed and stained with haematoxylin and eosin (H\&E) staining. Stained filters were then mounted on to slides and viewed by a consultant pathologist to assess for the presence of CTCs. All patient samples were processed using the ScreenCell device and scored as being either negative or positive for CTCs, based on the following characteristics, large epithelioid cells with, nuclear enlargement and an increased nuclear to cytoplasmic ratio.

All recruited patients were diagnosed with NSCLC, of which $21(64 \%)$ patients were diagnosed with adenocarcinoma and $12(36 \%)$ patients with squamous cell carcinoma. The median age of the patients was 64 years, with an interquartile range (IQR) of 13.7 years. Fifteen $(45 \%)$ patients were male. Fifteen $(45 \%)$ patients had stage I lung cancer, eleven $(33 \%)$ patients had stage II, $6(15 \%)$ patients had stage III and $1(3 \%)$ patient had stage IV disease (T3N2M1a) due to a metastatic pleural deposit at the time of surgical resection.

Twenty-eight (85\%) patients had a lobectomy, $1(3 \%)$ patient required pneumonectomy, and $4(12 \%)$ patients who were not fit enough for a lobectomy had a wedge resection. Surgery was performed thoracoscopically in $7(21 \%)$ patients and with an open approach in $26(79 \%)$ patients (25 thoracotomies, 1 sternotomy due to bilateral lesions).

Statistical analysis was calculated on GraphPad Prism version 5 (GraphPad Software, Inc.), and the survival proportion was analysed as a Kaplan-Meier plot. Statistical significance was calculated using a Chi-square test, and a hazard ratio (HR) was calculated with a $95 \%$ confidence interval (CI). The analysis between lung cancer stage and

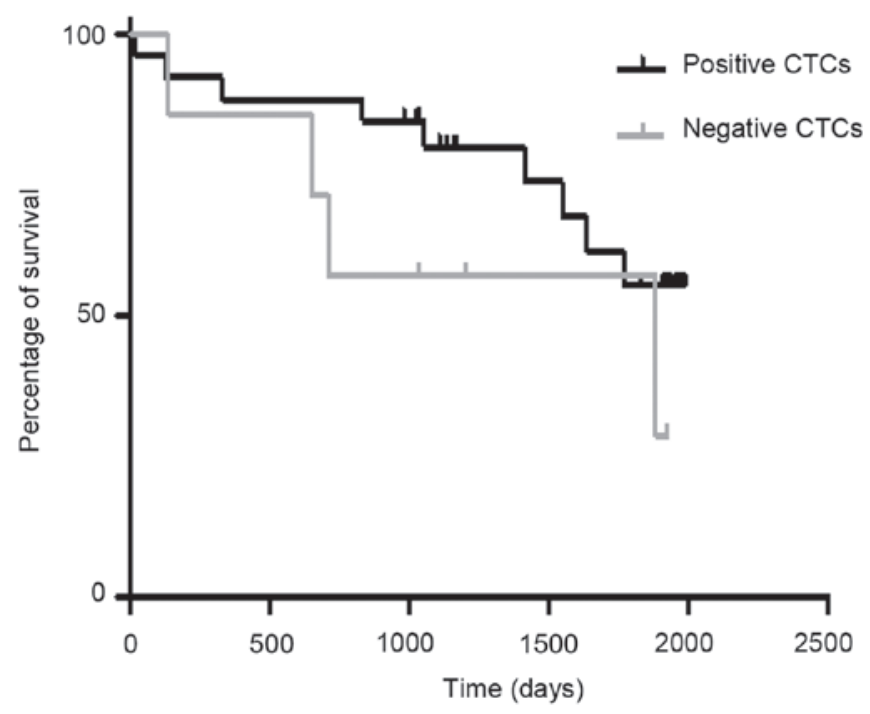

Figure 1. Kaplan-Meier plots of survival for patients positive and negative for CTCs. There was no relationship between positive CTCs and poorer survival (Chi-squared 1.47, $\mathrm{P}=0.23$; hazard ratio 0.42 , 95\% CI: 0.1-1.7). CTCs, circulating tumour cells; CI, confidence interval.

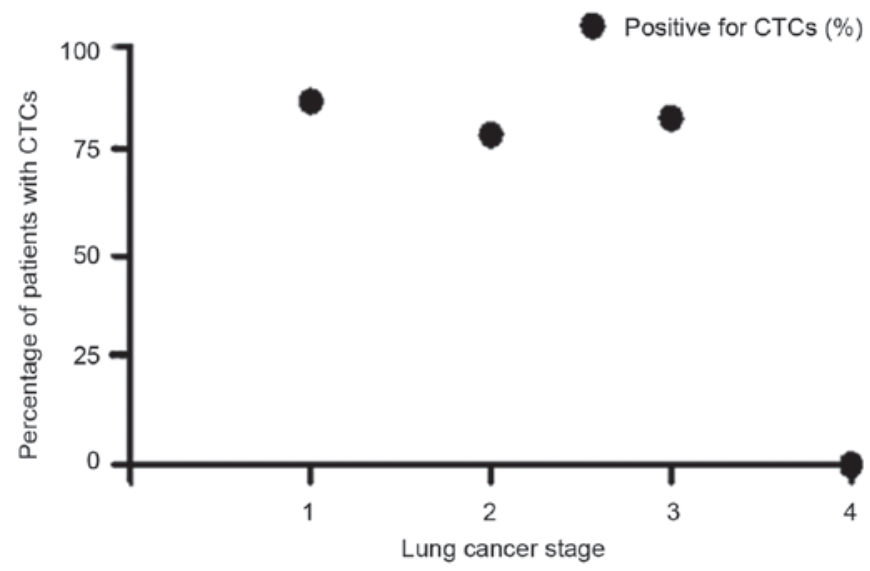

Figure 2. Pearson correlation of the percentage of patients with positive circulating tumours cells and lung cancer stage. Pearson's correlation coefficient $r=-0.80, P=0.20$. CTCs, circulating tumour cells.

the proportion of patients with positive CTCs was performed using Pearson's correlation. Tumour node metastasis version 7 was used for staging patients. Analysis between groups was performed using a two-sided Student's t-test. 
Table II. Survival depending on lung cancer stage and CTC status.

\begin{tabular}{|c|c|c|c|c|c|}
\hline \multirow[b]{2}{*}{ Stage } & \multirow[b]{2}{*}{ Total (\%) } & \multicolumn{2}{|c|}{ Total } & \multicolumn{2}{|c|}{ CTC status } \\
\hline & & Alive & Deceased & Detected & Not detected \\
\hline 1 & $15(45.4 \%)$ & $9(60 \%)$ & $6(40 \%)$ & $13(87 \%)$ & $2(13 \%)$ \\
\hline 2 & $11(33.3 \%)$ & $8(73 \%)$ & $3(27 \%)$ & $8(73 \%)$ & $3(27 \%)$ \\
\hline 3 & $6(18.1 \%)$ & $3(50 \%)$ & $3(50 \%)$ & $5(83 \%)$ & $1(17 \%)$ \\
\hline 4 & $1(3.3 \%)$ & $0(0 \%)$ & $1(100 \%)$ & $0(0 \%)$ & $1(100 \%)$ \\
\hline Total & 33 & $20(61 \%)$ & $13(39 \%)$ & $26(79 \%)$ & $7(21 \%)$ \\
\hline
\end{tabular}

There was no direct correlation between the stage and detection of CTCs. A Pearson's analysis between proportion of patients with positive CTCs status and lung cancer stage demonstrated a negative correlation, Pearson's $r=-0.80, P=0.20$. CTC, circulating tumour cells .

Table III. Median survival based on operative approach and CTC status.

\begin{tabular}{lccccc}
\hline & & \multicolumn{2}{c}{ CTC detected } & & CTC not detected \\
\cline { 3 - 5 } Surgery & Total & Total $(\%)$ & Survival (months) & & Total (\%) \\
\hline VATS & 7 & $6(86)^{\mathrm{a}}$ & $54^{\mathrm{b}}$ & $1(14)$ & Survival (months) \\
Open & 26 & $20(77)^{\mathrm{a}}$ & $38^{\mathrm{b}}$ & $6(23)$ & $23^{\mathrm{b}}$ \\
\hline
\end{tabular}

${ }^{\mathrm{a}}$ No significant difference was observed in the proportion of patients in the VATS or open group who were positive for CTCs, $\mathrm{P}=0.63$. ${ }^{\mathrm{b}} \mathrm{Survival}$ in the patients undergoing a thoracoscopic approach was improved but not significantly, $\mathrm{P}=0.43$. CTC, circulating tumour cells.

Table IV. Median survival based on gender and CTC status.

\begin{tabular}{lccccc}
\hline & & \multicolumn{2}{c}{ CTC detected } & & CTC not detected \\
\cline { 3 - 4 } Sex & Total & Total $(\%)$ & Survival (months) & & Total (\%) \\
\hline Female & 18 & $14(43)$ & $58^{\mathrm{a}}$ & $4(12)$ & $37^{\mathrm{a}}$ \\
Male & 15 & $12(36)$ & $38^{\mathrm{b}}$ & $3(9)$ & $21^{\mathrm{b}}$ \\
\hline
\end{tabular}

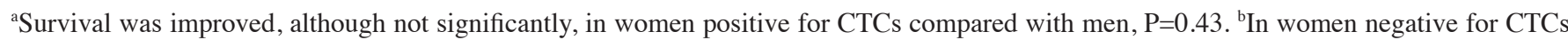
survival was longer, compared to men, but this was notindicated to be significant, $\mathrm{P}=0.61$. CTC, circulating tumour cells.

\section{Results}

CTCs were detected in $26(79 \%)$ patients. At the end of our follow-up period at a median of 5 years, 20 patients (61\%) were still alive (Table I). In patients who were positive for CTCs 9 out of 26 patients ( $35 \%$ ) had succumbed to the disease, whereas in patients negative for CTCs 4 out of 7 (57\%) patients had succumbed to the disease $(\mathrm{P}=0.29)$. Of the 13 patients who succumbed to the disease $9(69 \%)$ had CTCs detected. Whereas in the 20 patients who were still alive $17(85 \%)$ had detected CTCs.

Overall survival was analysed using a Kaplan Meier plot (Fig. 1) of patients positive and negative for CTCs. It showed no relationship between positive CTCs being associated with poorer survival, Chi-squared $1.47, \mathrm{P}=0.23, \mathrm{HR}=0.42$ (95\% CI: 0.1-1.7). Median survival in patients with CTCs was 46.5 months whereas it was only 34.1 months in patients without CTCs.
There was no direct correlation between the stage and detection of CTCs (Table II). A Pearson's analysis (Fig. 2) between proportion of patients with positive CTCs status and lung cancer stage showed negative correlation, Pearson's $\mathrm{r}=-0.80, \mathrm{P}=0.20$.

Of the 21 patients with adenocarcinoma $17(81 \%)$ patients had positive CTCs whereas of the 12 patients with squamous cell carcinoma $9(75 \%)$ patients had positive CTCs $(\mathrm{P}=0.70)$ (Table I).

A total of 6 of the $7(86 \%)$ patients that had their operation performed thoracoscopically were positive for CTCs whereas 20 of the $26(77 \%)$ patients that had an open operation were positive for CTCs $(\mathrm{P}=0.63$; Table III). Survival in the patients undergoing a thoracoscopic approach was 51 vs. 38 months in the open group $(\mathrm{P}=0.43)$.

Survival was better, although not significantly, in women positive for CTCs compared with men (58 vs. 38 months, 
respectively), $\mathrm{P}=0.43$. In women negative for $\mathrm{CTC}$ s survival was 37 months compared to 21 months in men negative for CTCs ( $\mathrm{P}=0.61$; Table IV).

In the subgroup of patients who underwent a wedge resection 3 of the 4 patients $(75 \%)$ were positive for CTCs, whereas in the group undergoing lobectomy or pneumonectomy 23 out of 29 patients $(79 \%)$ were positive for CTCs $(\mathrm{P}=0.85)$.

\section{Discussion}

Previously we demonstrated the ability of ScreenCell in detecting CTCs in patients with lung cancer (8). In this study we sought to evaluate the impact of positive CTCs on long term follow-up. There was no effect of the presence of CTCs on median survival, in fact their presence was actually surprisingly associated with longer median survival compared to those patients negative for CTCs, although not significantly. This was also the case when we analysed the results with shorter follow up and fewer patients (8).

It is known that different types of lung cancer metastasize via difference means and this can reflect their potential to spread. Small cell lung cancer and undifferentiated NSCLC spread via single cell or small cell cluster movement whereas in adenocarcinoma and squamous cell carcinoma spread results from movement of large clusters of organised cells (13). ScreenCell looks for individual CTCs. Given that all patients in our cohort had either adenocarcinoma or squamous cell carcinoma it may be that the presence of isolated CTCs is not clinically relevant to the metastatic potential as these tumour will not metastasize until organised clusters of cells are formed. In our cohort there was no difference in the proportion of CTCs between adenocarcinomas and squamous cell carcinomas however this likely reflects that they both metastasize in a similar way (13). The improved prognosis (although not significant) in patients with CTCs may reflect the fact that detection of single CTCs primes the immune system to respond to organised clusters of cells.

Our data did not show a significant association between the proportion of patients with CTCs and lung cancer stage (Fig. 2). However it is interesting to note that even in patients with stage I lung cancer $87 \%$ of patients had CTCs found in a sample of peripheral blood. Thus even at this early stage lung cancers are producing CTCs which can be detected in a peripheral blood sample. This highlights the potential importance that the detection of CTCs could have as a screening test for early stage lung cancer.

There is evidence to support that patients undergoing a VATS lobectomy for early stage lung cancer have improved survival compared to those patients undergoing an open operation. One potential reason for this is that there is reduced manipulation of the lung and the tumour with a VATS approach compared to open surgery, which reduces the number of CTCs shed during surgery $(14,15)$. However we were unable to detect any difference in the number of CTCs between VATS and open surgery to support this theory although the numbers in our VATS group were small.

Limitations of our study included the small sample size of 33 patients and the heterogeneity of the cohort with patients with different lung cancer stage. The study was designed as a pilot study to assess whether a link exists between ScreenCell detected CTCs and prognosis. Although there was heterogeneity in the cohort it was designed to reflect clinical practice of patients operated on for lung cancer.

Our result show that the presence of CTCs detected with ScreenCell does not have an effect on prognosis in patients with operated NSCLC. We believe that this may reflect the fact the ScreenCell detects single cells which may not be relevant to the metastatic potential of lung adenocarcinoma and squamous cell carcinoma.

There was no relationship between lung cancer stage and the proportion of patients with CTCs. It is interesting that even in stage I lung cancers more than $80 \%$ of patients were found to have detectable CTCs which is encouraging in supporting this technology to aid early lung cancer diagnosis.

\section{Acknowledgements}

Not applicable.

\section{Funding}

The present study was supported by funding was from The Harefield Charitable Cryotherapy Trust Funds.

\section{Availability of data and materials}

All data generated or analysed during this study are included in this published article.

\section{Authors' contributions}

JB wrote the manuscript, analysed the data and performed the statistical tests. DC collected and analysed the data and helped with writing the manuscript. AR analysed the samples for the presence of circulating tumour cells, EK was involved in the designing the study and writing the manuscript. VA designed the study and wrote the manuscript.

\section{Ethics approval and consent to participate}

Ethical approval was sought prior (ethical approval no. 10/H0504/9), with consent obtained pre-operatively. Patients who participated in this research had complete clinical data. The signed informed consents were obtained from the patients or the guardians.

\section{Patient consent for publication}

Not applicable.

\section{Competing interests}

The authors declare that they have no competing interests.

\section{References}

1. Lung cancer statistics. Cancer research UK, Oxford, 2018. https://www.cancerresearchuk.org/health-professional/cancerstatistics/statistics-by-cancer-type/lung-cancer. Accessed April 4, 2018 
2. Bannister N and Broggio J: Survival for people diagnosed by cancer type, split by stage at diagnosis. Data based on people diagnosed in England in 2012, 2013 and 2014. Produced in collaboration with Public Health England (PHE). In: Cancer survival by stage at diagnosis for England (experimental statistics): Adults diagnosed 2012, 2013 and 2014 and followed up to 2015. Office for National Statistics, Newport, pp1-30, 2016.

3. National Lung Screening Trial Research Team, Aberle DR, Adams AM, Berg CD, Black WC, Clapp JD, Fagerstrom RM, Gareen IF, Gatsonis C, Marcus PM and Sicks JD: Reduced lung cancer mortality with low dose computed tomographic screening. N Engl J Med 365: 395-409, 2011.

4. Pantel K, Brakenhoff RH and Brandt B: Detection, clinical relevance and specific biological properties of disseminating tumour cells. Nat Rev Cancer 8: 329-340, 2008.

5. Zhang Z, Ramnath N and Nagrath S: Current Status of CTCs as Liquid Biopsy in Lung Cancer and Future Directions. Front Oncol 5: 209, 2015.

6. Hou HW, Warkiani ME, Khoo BL, Li ZR, Soo RA, Tan DS Lim WT, Han J, Bhagat AA and Lim CT: Isolation and retrieval of circulating tumor cells using centrifugal forces. Sci Rep 3: 1259, 2013.

7. Dent BM, Ogle LF, O'Donnell RL, Hayes N, Malik U, Curtin NJ, Boddy AV, Plummer ER, Edmondson RJ, Reeves HL, et al High-resolution imaging for the detection and characterisation of circulating tumour cells from patients with oesophageal, hepatocellular, thyroid and ovarian cancers. Int J Cancer 138: 206-216, 2016

8. Chudasama D, Barr J, Beeson J, Beddow E, McGonigle N, Rice A, Nicholson A and Anikin V: Detection of Circulating Tumour Cells and Survival of Patients with Non-small Cell Lung Cancer. Anticancer Res 37: 169-173, 2017.
9. Chudasama D, Rice A, Anikin V, Soppa G and Dalal P: Circulating Tumour Cells in Patients with Malignant Lung Tumors Undergoing Radio-frequency Ablation. Anticancer Res 35: 2823-2826, 2015.

10. Chudasama D, Burnside N, Beeson J, Karteris E, Rice A and Anikin V: Perioperative detection of circulating tumour cells in patients with lung cancer. Oncol Lett 14: 1281-1286, 2017.

11. Chudasama D, Rice A, Soppa G and Anikin V: Circulating tumour cells in patients with lung cancer undergoing endobronchial cryotherapy. Cryobiology 71: 161-163, 2015.

12. Freidin MB, Tay A, Freydina DV, Chudasama D, Nicholson AG, Rice A, Anikin V and Lim E: An assessment of diagnostic performance of a filter-based antibody-independent peripheral blood circulating tumour cell capture paired with cytomorphologic criteria for the diagnosis of cancer. Lung Cancer 85: 182-185, 2014.

13. Popper HH: Progression and metastasis of lung cancer. Cancer Metastasis Rev 35: 75-91, 2016.

14. Chen FF, Zhang D, Wang YL and Xiong B: Video-assisted thoracoscopic surgery lobectomy versus open lobectomy in patients with clinical stage I non-small cell lung cancer: A meta-analysis. Eur J Surg Oncol 39: 957-963, 2013.

15. Cai YX, Fu XN, Xu QZ, Sun W and Zhang N: Thoracoscopic lobectomy versus open lobectomy in stage I non-small cell lung cancer: A meta-analysis. PLoS One 8: e82366, 2013.

(i) () This work is licensed under a Creative Commons BY No ND Attribution-NonCommercial-NoDerivatives 4.0 International (CC BY-NC-ND 4.0) License. 